\title{
Coherent light transport in a cold Strontium cloud
}

\author{
Y. Bidel, ${ }^{1}$ B. Klappauf, ${ }^{2}$ J.C. Bernard, ${ }^{1}$ D. Delande, ${ }^{3}$ G. \\ Labeyrie, ${ }^{1}$ C. Miniatura, ${ }^{1}$ D. Wilkowski, ${ }^{1}$ 甩 and R. Kaiser ${ }^{1}$ \\ ${ }^{1}$ Laboratoire Ondes et Désordre, FRE 2302, \\ 1361 route des Lucioles F-06560 Valbonne, France \\ ${ }^{2}$ Now at: Optoelectronics Research Center, \\ University of Southampton, SO17 1BJ Southampton, United Kingdom \\ ${ }^{3}$ Laboratoire Kastler Brossel, Université Pierre et Marie Curie, F-75252 Paris, France
}

(Dated: October 28, 2018)

\begin{abstract}
We study light coherent transport in the weak localization regime using magneto-optically cooled strontium atoms. The coherent backscattering cone is measured in the four polarization channels using light resonant with a $J_{g}=0 \rightarrow J_{e}=1$ transition of the Strontium atom. We find an enhancement factor close to 2 in the helicity preserving channel, in agreement with theoretical predictions. This observation confirms the effect of internal structure as the key mechanism for the contrast reduction observed with an Rubidium cold cloud (see: Labeyrie et al., PRL 83, 5266 (1999)). Experimental results are in good agreement with Monte-Carlo simulations taking into account geometry effects.
\end{abstract}

PACS numbers: 42.25.Fx, 32.80.Pj

*Electronic address: wilkowsk@inln.cnrs.fr; URL: http://www-lod.inln.cnrs.fr/ 
During the past twenty years, the outstanding development of mesoscopic physics led to a critical inspection of coherent effects in wave transport. First motivated by electronic transport in conducting devices [1], the underlying physical ingredients proved to be relevant to any linear waves and in particular to light. This triggered active research in the field of optics during the past two decades [2] leading to the observation of coherent backscattering [3] and universal conductance fluctuations [4] to quote a few. A challenge in this field is still the observation of strong localization of visible light. It was recently reported for nearinfrared light using semi-conductors powders [5], but the interpretation of the experiment in term of Anderson localization was questioned [6]. Cold atoms have been quite recently considered as promising scattering media to achieve strong localization [7]. Indeed, they constitute perfectly monodisperse samples of resonant point-dipole scatterers with large cross-sections. Moreover high spatial density is achieved by adequate trapping techniques [8, 9].

In this letter we report the observation of coherent backscattering (CBS) of light on cold strontium atoms in the weak localization regime $k l \gg 1$ ( $k$ is the light wavenumber and $l$ the elastic mean free path). CBS is an interferential enhancement of the average scattered intensity reflected off a disordered scattering medium [10]. It originates from a twowave constructive interference (near exact backscattering) between waves travelling along a given scattering path and its reversed counterpart. For classical scatterers, bearing on general symmetry arguments valid in the absence of any magnetic field, the CBS interfering amplitudes have been shown to have equal weights at exact backscattering in the so-called parallel polarization channels [11]. In the lin\|lin channel the incoming and detected light fields have same linear polarization. In the $h \| h$ channel, both light fields are circularly polarized with the same helicity, that is opposite polarizations (because the CBS signal is emitted in the backward direction). In the perpendicular channels, nothing ensures the equality of the two interfering amplitudes and the contrast of the interference is decreased. The single scattering events require a separate treatment as the direct and reversed paths coincide and do not contribute to the CBS enhancement in the backward direction. For spherically symmetric scatterers, single scattering does not contribute in the $\operatorname{lin} \perp \operatorname{lin}$ and $h \| h$ channels. Thus, the CBS contrast (peak to background ratio) is predicted and has been observed to be exactly 2 in the helicity preserving polarization channel $h \| h$ [12]. Using an atomic gas at resonance, a dynamic breakdown of the CBS effect can occur due to the 
scatterers motion during the transit time of a photon inside the medium. This restricts the RMS velocity $\delta v$ below a critical velocity given by $v_{c}=\Gamma / k$ (where $\Gamma$ is the width of the atomic dipole resonance), a condition which is well fulfilled for a laser cooled atomic gas [13]. The quantum internal structure of atoms has also severe consequences for coherent light transport in atomic media. A degeneracy in the groundstate induces a dramatic scrambling of the CBS effect [14. This has been first experimentally observed with a cold Rubidium sample on a $J_{g}=3 \rightarrow J_{e}=4$ transition [13]. These results highly motivated the use of nondegenerate groundstate atoms, like strontium, to benefit from full interference effects in coherent transport.

The cold strontium ( $\mathrm{Sr}$ ) cloud is produced in a magneto-optical trap (MOT). The transverse velocity of an effusive atomic beam, extracted from a $500^{\circ} \mathrm{C}$ oven, is immediately compressed with a 2D optical molasse. A $27 \mathrm{~cm}$ long Zeeman slower then reduces the longitudinal velocity to within the capture velocity range of the MOT $(\sim 50 \mathrm{~m} / \mathrm{s})$. The Zeeman slower, molasses, MOT, and probe laser beams at $461 \mathrm{~nm}$ are generated from the same frequency-doubled source. Briefly, a single-mode grating stabilized diode laser and a tapered amplifier are used in a master-slave configuration to produce $500 \mathrm{~mW}$ at $922 \mathrm{~nm}$. The infrared light is then frequency doubled in a semi-monolithic standing wave cavity with an intra-cavity $\mathrm{KNbO}_{3}$ crystal. The cavity is resonant for the infrared light while the second harmonic exits through a dichroic mirror providing $150 \mathrm{~mW}$ of tunable single-mode light, which is then frequency locked on the $461 \mathrm{~nm}{ }^{1} \mathrm{~S}_{0^{-}}{ }^{1} \mathrm{P}_{1}$ strontium line in a heat pipe. We use acousto-optic modulators for subsequent amplitude and frequency variations. The MOT is made of six independent trapping beams of $5.2 \mathrm{~mW} / \mathrm{cm}^{2}$ each, red-detuned by $\delta=-\Gamma$ from the resonance. The saturation intensity is $42.5 \mathrm{~mW} / \mathrm{cm}^{2}$ and the natural width of the transition is $\Gamma / 2 \pi=32 \mathrm{MHz}$. Two anti-Helmoltz coils create a $100 \mathrm{G} / \mathrm{cm}$ magnetic field gradient to trap the atoms. A small population loss to metastable states is repumped to the ground state using two additional red lasers. The best achieved optical thickness of our Sr MOT is $b \approx 3$. It is deduced from transmission measurements of a resonant probe through the cloud shortly after switching the MOT off. Note that because the optical thickness of the atomic cloud is larger than one, the imaging of the cloud does not yield a signal proportional to the atomic density (flattening at the center) and the whole process thus overestimates the size of the cloud (see discussion below). The number of trapped atoms $N \simeq 10^{7}$ is derived from the MOT fluorescence signal. From a CCD image the RMS radius of the cloud has 
been estimated at $0.65 \mathrm{~mm}$ yielding a mean free path $l \approx 0.5 \mathrm{~mm}(k l \simeq 7000)$. The RMS velocity of the atoms is less that $1 \mathrm{~m} / \mathrm{s}$, well below the critical velocity $v_{c}=15 \mathrm{~m} / \mathrm{s}$.

The detailed experimental procedure for the CBS observation has been published elsewhere [13]. For the Sr experiment, the signal is obtained using a collimated resonant probe beam with a waist of $3 \mathrm{~mm}$. To avoid any effects linked to the saturation of the optical transition (non-linearities, inelastic radiation spectrum) [15], the probe intensity is weak (saturation parameter $s=0.02$ ). The scattered light is collected in the backward direction by placing a CCD camera in the focal plane of an achromatic doublet. The angular resolution of our apparatus is $0.1 \mathrm{mrad}$, roughly twice the CCD pixel angular resolution. To avoid recording the MOT fluorescence signal while recording the CBS signal, a time-sequenced experiment is developed. The trapping beams and the magnetic field gradient are switched off during the CBS acquisition sequence (duration $100 \mu s$ ) and then switched on to recapture the atoms (duration $95 \%$ of the $6 \mathrm{~ms}$ total cycle time). This procedure also eliminates any possible unwanted nonlinear wave mixing processes. The whole time sequence is then repeated as long as necessary for a good signal-to-noise ratio (typically 15 minutes in the experiment). During the CBS sequence, the image field is opened (and then closed during the MOT sequence) thanks to a mechanical chopper. During the CBS probe interaction time, each atom scatters about 200 photons on average but always remains in resonance since the mean atomic velocity increase is far below $v_{c}$. Consequently, most of them are recaptured during the following MOT sequence. The CBS images (see Fig.四) are finally obtained by subtracting the background image taken without cold atoms. This background image is recorded in the absence of the magnetic gradient during all the acquisition time. We thus checked that the fluorescence signal from the residual Sr atoms was negligible.

In the helicity preserving channel $(h \| h)$, the enhancement factor is found to be $\alpha=$ $1.86 \pm 0.10$ with an optical thickness of $b=2.9$ (see Fig. 2), slightly lower the theoretical prediction $\alpha=2$. Several experimental issues can explain the difference. First, the finite angular resolution of the detection apparatus lowers the CBS enhancement factor by an amount evaluated to $\delta \alpha \approx 0.06$. Because single scattering contributes more than $90 \%$ of the total signal in the two authorized channels (see Table $\mathbb{Q}$ ), the reduction of the cone contrast due to imperfect polarization channel isolation in the $h \| h$ is not negligible. We have measured, in the limit of low optical thickness where single scattering dominates over multiple scattering, the fraction of detected light in the forbidden $h \| h$ channel with respect 
to the total scattered light. We found a channel isolation about $5.10^{-4}$ leading to $\delta \alpha \approx 0.03$. Note that single scattering depolarization induced by stray magnetic field acts here like an imperfect polarization isolation. For this reason, its impact on the cone reduction has been minimized during the channel isolation procedure. Another possible source of contrast reduction is a Faraday effect induced by the residual magnetic field [16]. It turns out that, despite the huge Verdet constant in the atomic gas medium [17], its effect should be smaller than the previously discussed ones. We also checked that the finite transverse size of the laser beam has no significant influence on the signal. Taking into account the systematic errors, we find that the CBS enhancement factor should rather be $\alpha=1.91$, consistent with the measured value. A remaining (but yet uncontrolled) source of error in determining $\alpha$ is certainly an imperfect estimation of the background level measured at angles large compared to the cone width $\theta \gg \Delta \theta_{\mathrm{CBS}}$.

In the other polarization channels, we observe lower enhancement factors as predicted by the theory (see table 凹). In the $\operatorname{lin} \| \operatorname{lin}$ and $h \perp h$ channels, the small enhancement factors are mainly due to the strong single scattering contribution - see the relative incoherent background values given in table - - which is very important since the optical thickness is not very large. In the lin $\perp$ lin channel (where single scattering is absent), the relatively high contrast value is explained by the low optical thickness. Indeed, in this situation, short scattering paths dominate and double scattering is known to exhibit full interference contrast in all polarization channels [18]. In Table [, we also show data obtained with a Monte-Carlo (MC) calculation, where the amplitude of a multiple scattering path is computed as a function of the initial and final polarizations and of the geometrical positions of the various scatterers. We use a Gaussian distribution for the spatial density of scatterers and take into account the spatial variations of the mean free path during the photon propagation. Our numerical method is tantamount to computing the integral involved in the configuration average using a MC procedure. Given a spatial configuration of the scatterers, we compute simultaneously the various scattering contributions at different scattering orders using the "partial photon" trick [19]. Typically, it is enough to launch less than 1 million photons on the medium to get a good signal/noise ratio for the CBS peak. For all polarization channels, there is a good agreement for the cone height between experiments and $\mathrm{MC}$ simulations adjusted to take into account the polarization channel isolation and angular resolution effects.

The experimental values $\Delta \theta_{\mathrm{CBS}}$ of the FWHM CBS angular cone width are systematically 
higher (by a factor 1.4) that the ones given by the MC simulation using the measured optical thickness $b$ and size of the atomic cloud. As discussed above, our experimental procedure slightly overestimates the size of the cloud. Modifying the size of the cloud (keeping $b$ constant) results only in a global multiplication of the angular scale, keeping identical both the enhancement factor and the cone shape. We are thus inclined to think that the actual RMS radius of the cloud is $0.45 \mathrm{~mm}$ instead of $0.65 \mathrm{~mm}$. With this corrected value, we observe an excellent agreement between $\mathrm{MC}$ and experimental data in all polarization

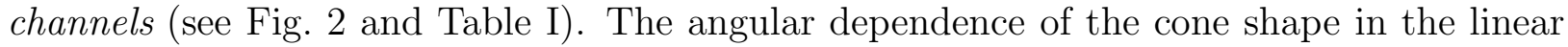
channels reflects the anisotropy of the scatterer's pattern [18. In the lin\|lin channel, an elliptical shape with major axis parallel to the incident polarization is predicted and indeed observed (Fig. 1 $\mathrm{k}$ ). In the lin $\perp$ lin channel, the directions of maximum scattering are tilted at $45^{\circ}$ from the incident polarization, yielding a "clover-leafed" CBS cone shape (Fig. 11d).

To summarize, we measured the coherent backscattering cone in four different characteristic polarization channels. Our results are in good agreement with a Monte-Carlo calculation. The restoration of a full interference contrast in coherent multiple scattering with atomic gases (as exemplified by the maximum enhancement factor of 2 obtained in the helicity preserving channel) has interesting potentialities for wave localization experiments with cold atoms. For example, in the quest for Anderson localization (which could be obtained only at high density where $k l \approx 1$ ) where interferences play a crucial role, a $J_{g}=0 \rightarrow J_{e}=1$ transition appears to be a good choice, since a degenerate internal structure is known to scramble the interference [14]. A maximum enhancement factor of 1.2 was found in $\mathrm{Rb}$ experiment 13]. Is it now possible to increase the cloud density to reach the Anderson localization threshold? For this purpose, cooling strontium with the intercombination line in a dipole trap appears to be a promising technique [8].

The authors thank the CNRS and the PACA region for their financial support. Laboratoire Kastler Brossel is laboratoire de l'Université Pierre et Marie Curie et de l'Ecole Normale Supérieure, UMR 8552 du CNRS.

[1] D.K. Ferry and S.M. Goodnick, Transport in Nanostructures, Cambridge University Press, New York (1997); S. Datta, Electronic Transport in Mesoscopic Systems, Cambridge Univer- 
sity Press, Cambridge (1995).

[2] V.L. Kuz'min and V.P. Romanov, Physics-Uspekhi 39, 231 (1996); M.C.W. van Rossum and Th.M. Nieuwenhuizen, Rev. Mod. Physics 71 , 313 (1999).

[3] P.E. Wolf and G. Maret, Phys. Rev. Lett. 55, 2696 (1985); M.P. Van Albada and A. Lagendijk, Phys. Rev. Lett. 55, 2692 (1985).

[4] F. Scheffold and G. Maret, Phys. Rev. Lett. 81, 5800 (1998).

[5] D.S. Wiersma, P. Bartolini, A. Lagendijk and R. Righini, Nature 390, 671 (1997).

[6] F. Scheffold, R. Lenke, R. Tweer, G. Maret, comment on the citewiersma97 paper, Nature 398, 207 (1999).

[7] Th. M. Nieuwenhuizen, A.L. Burin, Yu. Kagan and G.V. Shlyapnikov, Phys. Lett. A 184, 360 (1994).

[8] T. Ido, Y. Isoya and H. Katori, Phys. Rev. A 61, R061403 (2000); H. Katori, T. Ido, M. Gonokami, J. Phys. Soc. of Jap. 68, 2479 (1999).

[9] Ultracold Atoms and Bose-Einstein condensation, K. Burnett ed., OSA Trends in Optics and Photonics Series 7 (OSA, 1996).

[10] E. Akkermans, P.E. Wolf, R. Maynard and G. Maret, J. Phys. (Paris) 49, 77 (1988).

[11] B.A. van Tiggelen and R. Maynard, in Wave Propagation in Complex Media, IMA 96, edited by G. Papanicolaon (Springer, New-York), 252 (1997).

[12] D.S. Wiersma, M.P. van Albada, B.A. van Tiggelen and A. Lagendijk, Phys. Rev. Lett. 74, 4193 (1995).

[13] G. Labeyrie, F. de Tomasi, J.C. Bernard, C. Müller, C.A. Miniatura and R. Kaiser, Phys. Rev. Lett. 83, 5266 (1999); G. Labeyrie, C. Müller, D. Wiersma, C. Miniatura and R. Kaiser, J. Opt. B : Quantum Semiclass. Opt. 2 , 672 (2000).

[14] T. Jonckheere, C.A. Müller, R. Kaiser, C. Miniatura and D. Delande , Phys. Rev. Lett. 85, 4269 (2000); C. Müller, T. Jonckeere, C. Miniatura and D. Delande, Phys. Rev. A 64, 053804 (2001).

[15] V.M. Agranovich and V.E. Kravtsov, Phys. Rev. B 43, 13691 (1991); A. Heiderich, R. Maynard and B. van Tiggelen, Opt. Comm. 115, 392 (1995).

[16] R. Lenke and G. Maret, Eur. Phys. J. B 17, 171 (2000).

[17] G. Labeyrie, C. Miniatura and R. Kaiser, Phys. Rev. A 64, 033402 (2001).

[18] M.P. van Albada, B. van Tiggelen and A. Lagendijk, Phys. Rev. Lett. 58, 361 (1987). 
[19] R. Lenke and G. Maret, in Scattering in Polymeric and Colloidal Systems, edited by W. Brown and K. Mortensen (Gordon and Breach, Reading, 2000), p. 1-72. 
FIG. 1: Isocontours of the coherent backscattering cones obtained on a cloud on cold Strontium atoms in the four polarization channels. We plot the CBS signal after substraction of the incoherent background, as a function of the backscattering angle. All cone heights have been scaled to 1. For better signal/noise the image have been symmetrized. The total angular range is $1 \mathrm{mrad}$. The lowest isocontour correspond to roughly $20 \%$ of the peak intensity. For the linear channels, the incident polarization is horizontal.

FIG. 2: Angular scan of the CBS cone for the $h \| h$ polarization channel. The optical thickness is $b=2.9$. The experimental data are represented with open circles. For better signal/noise we perform an angular averaging of the original image. The solid line is the result of a MonteCarlo calculation taking into account the geometry of the atomic cloud (Gaussian distribution of the atomic density with variance $0.45 \mathrm{~mm}$ ) and experimental imperfections like the polarization channel isolation and angular resolution effects. The agreement is clearly excellent. The measured width of the cone is $\Delta \theta_{C B S}=0.50 \pm 0.04 \mathrm{mrad}$. 
TABLE I: Comparison between the CBS enhancement factor and peak width measured in the experiment with the results of a Monte-Carlo calculation, for optical thickness $b=2$. In each polarization channel, the experimental enhancement factor $\alpha$ is given with a $\pm 2 \sigma$ error bar. For linear polarization channels, the $\Delta \theta_{C B S}$ values are only given for scans parallel to the incident polarization. The results of MC simulation (noted MC) are given for a Gaussian distribution of the could with a variance $0.45 \mathrm{~mm}$. The experimental imperfections like the polarization channel isolation and angular resolution effects have been taken into account in the MC simulation values noted MC*. The "Background" column show the relative contribution of the channel of the total incoherent scattered intensity in the backward direction.

\begin{tabular}{|c|c|c|c|c|}
\hline Channel & & Background & $\alpha$ & $\Delta \theta_{C B S}(\mathrm{mrad})$ \\
\hline \multirow{3}{*}{$h \| h$} & Exp. & $7.5 \%$ & $1.77 \pm 0.13$ & $0.52 \pm 0.07$ \\
\hline & $\mathrm{MC}$ & $7.8 \%$ & 2 & 0.48 \\
\hline & $\mathrm{MC}^{*}$ & $7.8 \%$ & 1.87 & 0.52 \\
\hline \multirow{3}{*}{$h \perp h$} & Exp. & $92.5 \%$ & $1.17 \pm 0.03$ & $0.71 \pm 0.10$ \\
\hline & $\mathrm{MC}$ & $92.2 \%$ & 1.20 & 0.69 \\
\hline & $\mathrm{MC}^{*}$ & $92.2 \%$ & 1.19 & 0.75 \\
\hline \multirow{3}{*}{$\operatorname{lin} \| \operatorname{lin}$} & Exp. & $96.0 \%$ & $1.17 \pm 0.03$ & $0.9 \pm 0.2$ \\
\hline & $\mathrm{MC}$ & $95.5 \%$ & 1.24 & 0.92 \\
\hline & $\mathrm{MC}^{*}$ & $95.5 \%$ & 1.22 & 0.98 \\
\hline \multirow{3}{*}{$\operatorname{lin} \perp \operatorname{lin}$} & Exp. & $4.0 \%$ & $1.59 \pm 0.20$ & $0.5 \pm 0.3$ \\
\hline & $\mathrm{MC}$ & $4.5 \%$ & 1.74 & 0.48 \\
\hline & $\mathrm{MC}^{*}$ & $4.5 \%$ & 1.62 & 0.49 \\
\hline
\end{tabular}




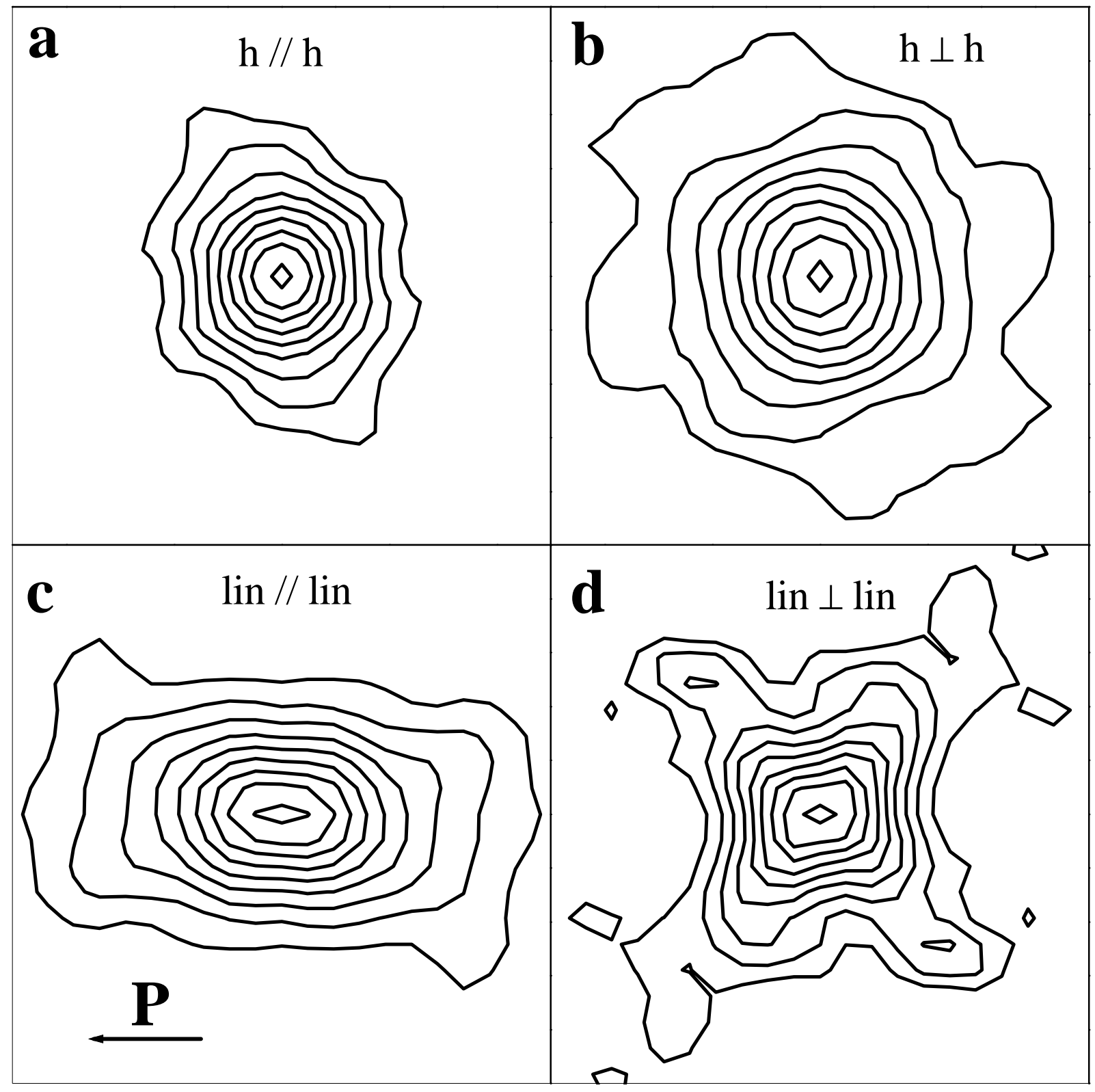




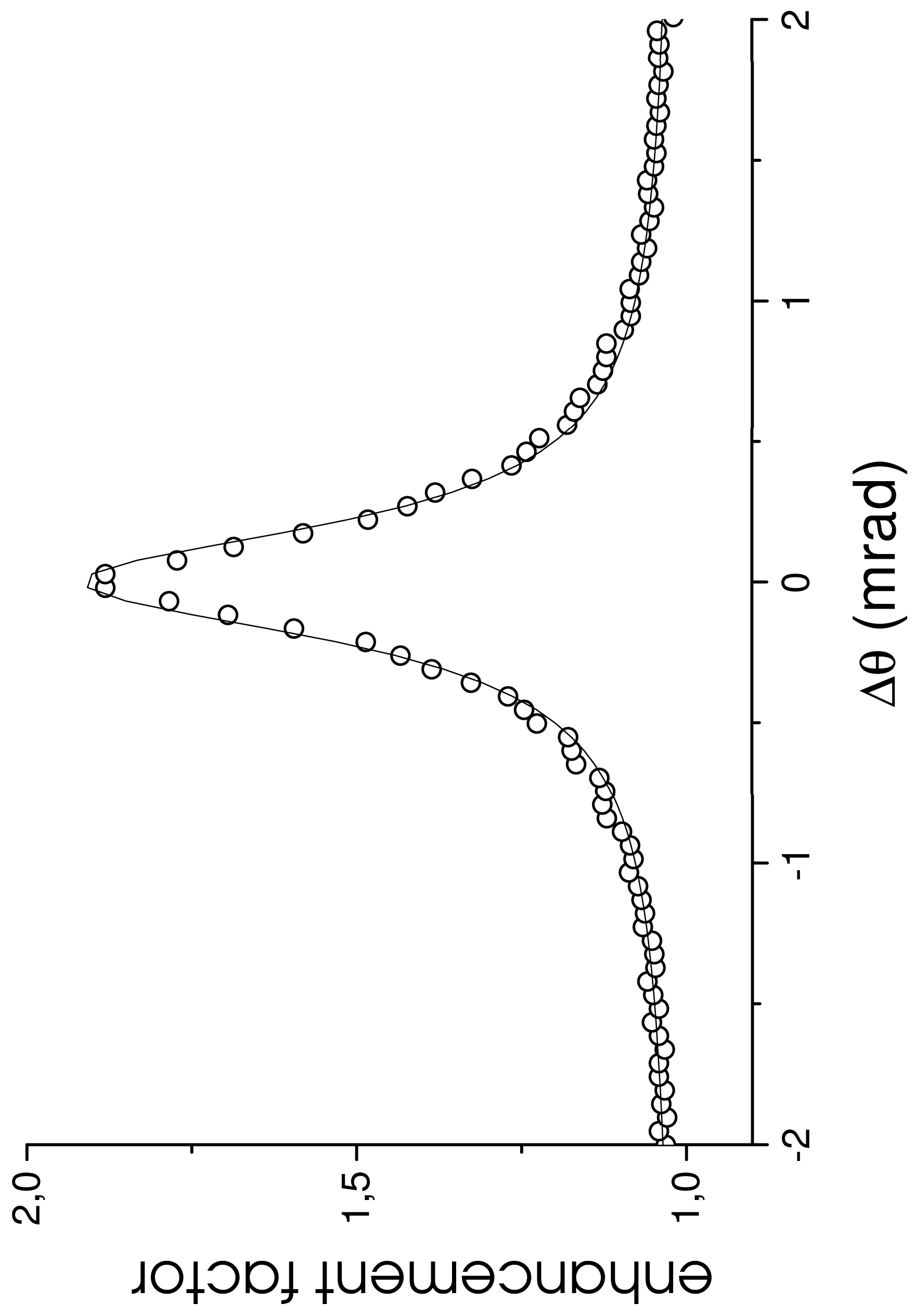

